STADLER SYMP. VOL. 6 (1974) University of Missouri, Columbia-9-

\title{
MOLECULAR CODING OF INFORMATION IN THE NERVOUS SYSTEM
}

\author{
G. UNGAR \\ Baylor College of Medicine \\ Houston, Texas 77025
}

SUMMARY

Evidence has been accumulating in the last ten years to support the existence of a molecular code for the processing of information by the nervous system. The most direct evidence has been provided by the behavioral bioassay approach which has led to the isolation of training-induced and behavior-inducing peptides (mnemones). The most probable role of these peptides is to establish and consolidate the connections of the neural circuits in which information is stored. Although this coding process is based on the genetically controlled labeling system by which neurons are organized into specific pathways, it must involve a step of non-genetic peptide synthesis.

\section{INTRODUCTION}

As a result of the revolution that has taken place in the biological sciences during the last quarter of a century, it is now obvious that the cycle and utilization of energy in living systems is controlled by genetic information stored in the DNA of the cell and by stimuli coming from the environment. While study of the processing of genetic information made rapid and spectacular advances, knowledge of the communication of living systems with their environment has been lagging behind. The main reason for this probably is the greater complexity of the problem. Processing of acquired information became, in the course of evolution, a function of the nervous system which is by far the most complex organizational network known to man. (The only type of acquired information that is handled independently from the nervous system is the immune process which will not be considered in this paper.) 
formation has been the reluctance to admit that, like genetic information, it may be coded in terms of molecular structure. The main purpose of this paper is to summarize the evidence that supports the existence of a molecular code in the nervous system and to show that it is in best agreement with the established facts.

\section{CODING IN THE NERVOUS SYSTEM}

The term code was borrowed from cryptography by information theory to designate any system of signals into which information can be converted. In this sense, languages, musical notations, geographic maps, etc., are all codes. I do not have to tell this audience how successful the introduction of this concept in. biology has been in elucidating the mechanism of heredity. The existence of a molecular code of genetic information has gone a long way towards explaining the processes by which specific and individual characters are transmitted throughout generations and which also provides a plausible mechanism for evolution. The genetic code contains instructions on how to put together the chemical constituents of a living system. We still do not know exactly how the morphological and functional characteristics of the organism are determined but there is little doubt that these are all inscribed in the code.

The innate organization of the nervous system, its specific sensory and motor pathways, are genetically determined. Apart from some maturation processes, this organization is completed in most species at birth. It provides the newborn organism with a number of prewired, genetically programmed pathways that serve the automatic control mechanisms without which it could not survive, as well as the elementary reflexes and, in some species, some highly complex instinctive behavioral patterns.

The prewired pathways (protocircuits) represent a stuctural. code. This principle was first formulated in 1826 by Johannes MüLLER in his "law of specific energies" which would be more properly called today the law of specific information. The law states that a sensation is independent of the nature of the stimulus applied to a nerve and depends only on its central connections. Thus, any stimulus applied to the optic nerve is interpreted as light, to the acoustic nerve as sound, etc.

The process by which genetic information controls the development of specific pathways has been the object of many hypotheses. The most widely accepted theory today is the concept of "chemospecificity of pathways" proposed by SPERRY (1963). It postulates that the neurons that are to be connected to form a pathway "recognize" each other by their identical or complementary chemical labels. This chemical recognition system can be regarded as the ultimate refinement of the basic process of histogenesis by which cells of the same type tend to form homotypic aggregates (MOSCONA 1963). At 
the early stages of neurogenesis, the neuroblasts form homogeneous aggregates but at later stages of differentiation different brain structures carry different labels (GARBER AND MOSCONA 1972).

It would seem logical for this labeling system to be genetically controlled but it is difficult to imagine that each of the $10^{7}$ protocircuits of the human brain requires a distinct gene. ROBERTS and FLEXNER (1966) have proposed a hypothesis that might resolve the difficulty but it remains to be supported by experimental evidence.

Many innate stimulus-response situations and behavioral patterns are known to be under chemical control. Some of the neurotransmitters play a role in the emotional motivation of behavior and can have a more specific effect when they are injected directly into well-defined brain structures, as in the induction of eating and drinking behavior. At a somewhat higher level, innate behaviors are under the direct or indirect control of peptides and proteins. The brain centers that ultimately command behavior produce the peptide releasing factors that initiate the chain of reactions leading to behavioral expression. The hypothalamic peptides are neurosecretory products; they are released at nerve endings and act on other cells, either directly or after having gone through the hypophyseal portal system or the general circulation.

The innate structural code provides prewired circuits by means of which a limited number of stimuli can elicit stereotyped responses which in the course of evolution proved to have survival value. The actual information fed into the system by these stimuli is defined, on the one hand, by the anatomical connections of the sensory receptors stimulated and, on the other hand, by the pattern of the bioelectric phenomena into which the energy of the stimulus is transduced. These electrical signals are analogous to what in computers is called the coding scheme. It includes mostly quantitative information regarding the intensity of the stimulus (converted into frequencies) and its temporal characteristics. The quality of the sensation, as mentioned above, depends on the central connections of the sensory nerves involved.

\section{CODING OF ACQUIRED INFORMATION}

All the processes mentioned up to now can be assumed to be under genetic control; they can be explained by the prenatal organization of protocircuits. However, the mechanisms involved in the processing of acquired information must be assumed to be non-genetic, since, by definition, acquired information cannot be part of the genome. Although the ability to learn is inherited and the type of information that can be processed is genetically limited, the actual information acquired by each individual organism is not transmitted to its descendants by biological means.

Learning postulates what is commonly called "plasticity" 
in the nervous system, i.e., the capability to change connections, to make new pathways not inscribed in the genetic code, called metacircuits (BARBIZET 1968).

The mechanism of plasticity is still a matter of hypotheses but during the last decade the general tendency has been to consider it more and more in chemical terms. Under the influence of HYDEN's pioneering studies (1967) a number of laboratories have been looking for chemical correlates of learning and memory. Others have investigated the effect of inhibitors of nucleic and protein metabolism on these processes. A third approach, finally, has consisted in attempting to detect learning-induced synthesis of coded molecules by bioassay.

\section{I CHEMICAL CORRELATES OF LEARNING}

In 1959, HYDÉN proposed that bioelectric wave patterns could be transduced into RNA sequences. Using this as a working hypothesis, he found that neurons involved in learning showed a different RNA base composition from control neurons. He first interpreted these as indicating the synthesis of a new molecular species of messenger RNA, presumably to be translated into the corresponding proteins. These findings are probably best interpreted today as indicating increased synthesis of messenger RNA from pre-existing derepressed DNA sites.

Increased RNA synthesis with or without changed base composition in correlation with learning was observed by several other laboratories (reviews by GLASSMAN 1969, BOOTH 1970). In recent years, however, the emphasis has shifted from RNA to proteins which are, after all, as Hyden himself recognized, the "executive molecules." Learning was shown to be correlated with increased incorporation of labeled amino acids into brain proteins, with increases in some glycoprotein fractions and in a brain-specific protein called S-100 protein.

The main difficulty in evaluating these experiments has been the distinction between chemical changes actually induced by learning and those associated simply with the increased neural activity that is a necessary concomitant of the learning process. Another drawback of the approach, as it is practiced by most laboratories, is that it may fail to detect the synthesis in the brain of small quantities of specific substances. It is, somehow, like an attempt at isolating specific hormonal agents from tissues by purely chemical methods without regard to their biological activity.

\subsection{ACTION OF METABOLIC INHIBITORS}

One way of checking whether RNA and protein synthesis was an essential correlate of learning has been to inhibit these processes and see whether inhibition had an effect on learning. This was done with inhibitors of DNA to RNA transcription such as 8-azaguanine and actinomycin D and 
with the inhibitors of RNA to protein translation, puromycin and cycloheximide. The most convincing results have probably been obtained with the latter drug because puromycin has some side-effects that may affect performance rather than learning and actinomycin $D$ is too toxic to allow long-term experiments (reviews by COHEN 1970, SQUIRE and BARONDES 1972).

Although the interpretation of these experiments is far from being simple, it is probable that metabolic inhibitors impair the process of consolidation of learned information, that is, the step that converts short-term memory into longterm memory. Under certain conditions there may also be inhibition of the retrieval of stored information. This approach can be criticized on the same grounds as the search for chemical correlates. They may just inhibit the increased neural activity necessary for learning without having an effect on some specific chemical processes involved in it.

\subsection{THE BEHAVIORAL BIOASSAY}

Detection of minute amounts of substances of unknown composition present in complex mixtures has traditionally been accomplished by biological assays. This is the method that has allowed the discovery of antibodies, hormones, vitamins, neurotransmitters and other biologically active substances.

The principle of the method as applied to the problem of neural coding is to communicate information to animals, i.e., train them for a specific behavior. If the training is accompanied by the synthesis of some coded substances, these could be detected by injecting recipient animals with brain preparations taken from the trained donors and testing the recipients for a behavioral change similar to the one induced in the donors by training.

Such experiments were started in 1962 in planarian worms and extended in 1965 to mammals. In spite of the controversy that developed over the validity of the method, up to the present 36 laboratories have published successful behavioral bioassays. Since the problem has been reviewed repeatedly (UNGAR 1971, UNGAR and CHAPOUTHIER 1971, several chapters in FUERDINGSTAD 1971 and ZIPPEL 1973), I shall mention only the use of the bioassay for isolating and identifying the active substances.

\section{TRAINING-INDUCED AND BEHAVIOR-INDUCING PEPTIDES (MNEMONES)}

Bioassays have been used for many years to isolate and chemically identify biologically active substances. The fractions obtained in the purification procedures are tested so as to select the one that contains the material to be isolated. This fraction is then further purified until the biological activity can be shown to be associated with a single homogeneous molecular species. 


\subsection{SCOTOPHOBIN}

Our laboratory started in 1968 to isolate the active substances extracted from brains taken from rats trained to avoid the dark. Innately, rats, like most rodents, show a distinct dark preference: when they have the choice between a lighted and a dark enclosure, they invariably choose the latter. This behavior can, however, be reversed if the animals are submitted in the dark to some painful stimulus, such as electric shocks. Extracts of brain taken from donor rats that have undergone such a training, when injected into untrained recipient animals, reproduce the dark avoiding behavior of the donors (UNGAR et al. 1968).

Over 4000 donor rats were trained over a period of two years and their brains were subjected to isolation procedures. Details of the purification have been published (UNGAR et al. 1972). About $300 \mathrm{ug}$ of a pure substance were obtained presenting all the characteristics of a peptide. The amino acid composition and sequence were determined and the peptide named scotophobin was reproduced by synthesis. The synthetic peptide was distributed to a number of laboratories, most of which confirmed our results (MALIN and GUTTMAN 1972, BRYANT et al. 1972, THINES et al. 1973).

Once the structure of a substance is determined, it is usually possible to devise a chemical method for its detection and thus bypass the bioassay. We were able to develop a technique for estimating scotophobin in the brain, by converting the peptide into a fluorescent dansyl derivative. Partially purified brain extracts can be dansylated and their constituents separated by two-dimensional thin-layer chromatography so that scotophobin can be estimated by densitometry of the corresponding spot.

By this method, we were able to measure the formation of endogenous scotophobin in rat and mouse brains during dark avoidance training. It was also possible to follow the fate of exogenous scotophobin in the brain after intraperitoneal injection (UNGAR 1973). Current work deals with the regional and subcellular distribution of both endogenous and injected scotophobin. Preliminary experiments have shown that about $2 / 3$ of the scotophobin formed during training is in the cortex but measurable amounts of it are distributed throughout the whole brain.

\subsection{AMELETIN}

In 1965, we found that injection of brain extracts taken from rats habituated to a sound stimulus, i.e., having lost their startle responses to it, causes loss of startle responses in the recipient animals (UNGAR and OCEGUERA-NAVARRO 1965). The active substance proved to be a hexapeptide (UNGAR and BURZYNSKI 1973) that was given the name ameletin (from the Greek ameleteos = indifferent). Reproduced by synthesis, this peptide gave only a partial effect when injected into re- 
cipient animals. A cyclic variant of ameletin is now being synthesized and will be tested.

\subsection{PEPTIDES EXTRACTED FROM GOLDFISH BRAIN}

More recently we started using goldfish as experimental animals because of their low cost of purchase and maintenance, and the possibility of keeping large numbers of them in a limited space.

Groups of goldfish were trained for avoidance behavior based on color discrimination in a tank divided into two compartments, one lighted in blue, the other in green. One group was trained to avoid the blue by being given electric shocks in the blue compartment; the other group was similarly trained to avoid the green. At completion of the training, extracts were prepared from the brains of both groups. When injected into untrained recipients, these extracts reproduced the avoidance of the donors (UNGAR et al. 1972). After having trained about 10,000 fish of each group, we purified the brain extracts by methods similar to the ones used in the isolation of scotophobin and obtained two peptides. The blue- : avoidance inducing peptide is now pure and awaits amino acid analysis. The peptide causing green avoidance still requires further purification.

A third peptide has been isolated from the brain of goldfish that we trained to adapt their swimming behavior to a float attached to them (SHASHOUA 1968, HELTZEL et al.1973). This learned motor adaptation is different from the other acquired behaviors used up to now and the peptide formed during its acquisition is larger than the others: it has about 20 to 25 amino acid residues. Purification of this peptide has been completed but its structure remains to be determined.

\subsection{DEFINITION OF A MNEMONE}

The term mnemone was coined to designate substances that are formed in the brain as a result of acquired information. They are different from hormones and other regulatory agents by the fact that they do not derive directly from the genetic code. Their formation raises the problem of a non-genetic mechanism for peptide synthesis. The advances of molecular biology have for a while invalidated all the previous mechanisms proposed for the synthesis of peptide bonds. In recent years, however, there has been a revival of interest in the possibility of a non-ribosomal synthesis of peptides. This seems to take place for small peptides like glutathione (MEISTER 1973) and in prokaryotes, at least, for longer chains like gramicidin (LIPMANN 1971). Recent evidence points to the presence in the brain of enzymes that could synthesize peptides by a non-genetic mechanism (REICHELT and KVAMME 1973, REICHLIN and MITNICK 1973). Whether mnemones are formed by some such mechanism or by a selective process, as is assumed for antibodies, is not clear at present. 


\section{MOLECULAR HYPOTHESES FOR NEURAL CODING}

There have been many allusions in the older literature to the possibility of a chemical mechanism in the storage of memory but no plausible hypothesis could be formulated until the chemistry of the nervous system became better known and, especially, until the emergence of a molecular code for hereditary information. The hypothesis of KATZ and HALSTEAD (1950) proposed "nucleoproteins" as the molecular carriers of memory and, through HYDEN, had considerable influence on those who attacked the problem experimentally.

On the whole, the many speculations published in the last two decades can be classified into a) "non-neurological" hypotheses, b) assumption of wave-pattern induced molecular changes and c) "sign-post" or "labeled metacircuit" hypotheses.

\subsection{NON-NEUROLOGICAL HYPOTHESES}

When the first evidence for molecular coding appeared, it was interpreted by some as a vindication of the so-called "field" or "mass"theory of brain function. This theory, originated in LASHLEY's negative findings on the localization of memory in the brain (1950), negated the importance of specific synaptic connections and emphasized the propagation of electrical pulses across masses of brain tissue. It seemed possible that memory traces, instead of being stored in terms of brain circuits, might be coded in molecular structures independently of synaptic connections. The molecular hypotheses of LANDAUER (1964) and ROBINSON (1966) assume that coded molecules may be synthesized in random nerve cells. McCONNELL (1965) tends to assume that molecular carriers of acquired information, like those of genetic information, may be present not only in nerve cells but in all cells of the body. This type of hypothesis is at present incompatible with most of the established notions on the functioning of the nervous system.

\subsection{TRANSDUCTION OF ELECTRICAL PULSES INTO MOLECULAR STRUCTURES}

The original hypothesis of KATZ and HALSTEAD (1950), mentioned above, was based on the assumption that information carried in the wave forms of the nerve impulses can be converted into molecular structures of nucleoproteins. HYDÉN (1959) accepted this assumption and tried to prove that the electrical pulses acted on RNA, producing new molecular species. When this idea clashed with the "central dogma" of molecular biology, the emphasis shifted to the possible derepression of parts of the genome that had not been transcribed before learning had taken place.

That neural activity can cause chemical changes in the neurons is a plausible assumption. The ionic environment of the cell has a definite influence on the enzymic reactions of cell metabolism and it is well known that $\mathrm{Na}^{+}, \mathrm{K}^{+}$and $\mathrm{Ca}^{++}$ ions undergo rapid changes in neural activity. The most ques- 
tionable postulate of these hypotheses is that the wave form carries all the information to be encoded as memory. There is no evidence at present that would indicate this; the wave patterns have no meaning outside the context of the pathways on which they travel and they probably contain only quantitative information.

\subsection{LABELING OF METACIRCUITS}

The idea that the molecular code may be superimposed on the structural code originated probably with SZILARD (1964). He distinguished two types of nerve cells: the "congenitally determined" neurons and the "memory" neurons: the former make up the innately connected protocircuits, while the latter are initially uncommitted but form the new connections between the innate pathways to create the metacircuits. This would take place by the process called "transprinting", i.e., the uptake by the memory cells of the "specific membrane proteins" by which the congenitally determined neurons are labeled.

The same assumptions have been made in other similar hypotheses (ROSENBLATT 1967, BEST 1968). The working hypothesis of my laboratory, first published in 1968, postulates that the molecular code is essentially a labeling of the synaptic connections correlated with the reprogramming process that results in the formation of metacircuits. It interprets transprinting as a combination of the innate labels of each of the protocircuits that participate in the formation of the new metacircuits. The mnemones would, therefore, represent a combination of two or more of the innate labels (UNGAR 1968, 1970, 1972).

The process by which mnemones are formed implies that substances can pass from one neuron to the other across the synaptic gap. Evidence for transsynaptic transport has been accumulating in recent years (GRAFSTEIN 1971). The hypothesis also postulates that peptide fragments can be combined together to form a new molecular species by a non-genetic, presumably enzymic process. This point was discussed above.

\subsection{PROPOSED MECHANISM OF MOLECULAR CODING}

The evidence available at present suggests that the coding process may include the following steps:

1. Activation of the protocircuits involved in the acquisition of new information. The resulting neural activity stimulates synthesis of the innate labels of these pathways, together with that of other proteins necessary for increased function. This may be the step that is blocked by metabolic inhibitors.

2. Simultaneous firing of two or more protociruits by increasing the permeability of synaptic membranes allows the transsynaptic transfer of the labels (transprinting). At the same time, the peptide-synthesizing enzymes are activated, probably in some specialized cells (memory neurons) to combine the innate labels and form a mnemone. 
3. The newly formed peptide is incorporated into the activated synaptic membranes to consolidate the connections. As long as these persist and the metacircuit can be reactivated, the information can be retrieved by the appropriate stimuli. The mechanism by which memory may be stored for many years would require a process of replication of the mnemones which is not clear at present.

In its present form, the hypothesis postulates that the neural code is based on the twenty-letter alphabet of the common amino acids. Combinations of these form the "vocabulary" of the code consisting of the genetically determined labels of the protocircuits. In the human brain the number of these is estimated to be of the order of $10^{7}$ which would not require sequences of more than six amino acids $\left(20^{6}=\right.$ $6 \times 10^{7}$ ). According to the process just summarized, these "words" can form a practically infinite number of "sentences" or "composite words" represented by the mnemones.

\section{CONCLUDING REMARKS}

Whether the hypothesis just summarized can withstand the test of experimental verification or not, the facts observed in the last ten years require an explanation. A segment of opinion holds that molecular coding is an "unnecessary" hypothesis and that the neural circuitry and its electrical impulse patterns are adequate to explain all the problems of brain function (BARONDES 1972). About the only chemical step admitted is the chemical mediation of synaptic transmission. Neurobiological thinking has, for the last twenty years, been dominated by the neurotransmitter concept which, when it emerged in the $1920^{\prime} \mathrm{s}$, was considered just as heretical as the molecular coding idea is today and did not gain recognition until the late $1940^{\prime} \mathrm{s}$.

There is no doubt that neurotransmitters play a role in information processing but at a level different from the mnemones. They are merely the chemical switches of the computer while the mnemones are the agents by which it is programmed and can reprogram itself. The possibility cannot be excluded that the mnemonal system derives from a primitive coding represented by the neurotransmitters. It is noteworthy that all the recognized neurotransmitters are either amino acids (glycine, glutamic acid) or derive from these by decarboxylation (serotonin, $\gamma$-amino butyric acid, dopamine) with additional hydroxylation (norepinephrine) or more complex transformations (acetylcholine). It is probable that as the complexity of the nervous system was increasing, single amino acids and their derivatives were less and less able to code for all the pathways and the task had to be taken over by peptide chains.

Explanation of learning and memory by non-specific chemical changes involving the machinery for synthesizing and releasing transmitters cannot account for the formation of metacircuits, their stability and specificity. Non-spe- 
cific interpretations do not require the existence of mnemones, which are therefore often interpreted merely as agents produced by the stressful stimuli associated with training. Resolution of the controversy will come with the isolation and characterization of more mnemones confirming their informationspecificity and with further studies elucidating the mechanism by which they contribute to the processing of information by the brain.

\section{ACKNOWLEDGEMENTS}

Most of the experimental work summarized in this paper was supported by grants from the National Institute of Mental Health and the National Institute of Education.

\section{LITERATURE CITED}

BARBIZET, J. 1968. Learning and use of knowledge. In: Neurosciences Research (Ehrenpreis, S. and O. C. Solnitsky, eds.) Pp. 315-332. Academic Press, New York.

BARONDES, S. H. 1972. Memory transfer. Science 176:631-632.

BEST, R. M. 1968. Encoding of memory in the neuron. Psychol. Rep. $\quad 22: 107-155$.

BOOTH, D. A. 1970. Neurochemical changes correlated with learning and memory retention. In: Molecular Mechanisms in Memory and Learning (Ungar, G., ed.) Pp. 1-57. Plenum Press, New York.

BRYANT, R. C., N. N. SANTOS and W. L. BYRNE 1972. Synthetic scotophobin in goldfish: specificity and effect on learning. Science 177:635-636.

COHEN, H. D. 1970. Learning, memory, and metabolic inhibitors. In: Molecular Mechanisms in Memory and Learning (Ungar, G., ed.) Pp. 59-70. Plenum Press, New York.

FJERDINGSTAD, E. J., ed. 1971. Chemical Transfer of Learned Information. North-Holland Publishing Company, Amsterdam. $268 \mathrm{pp}$.

GARBER, B. B. and A. A. MOSCONA. 1972. Reconstruction of brain tissue from cell suspensions. I and II. Devel. Biol. 27:217-234 and 235-243.

GLASSMAN, E. 1969. The biochemistry of learning. Ann. Rev. Biochem. $38: 605-646$. 
GRAFSTEIN, B. 1971. Transneuronal transfer of radioactivity in the central nervous system. Science 172:177-179.

HELTZEL, J. A. and G. UNGAR 1973. Purification from goldfish brain of two peptides coding for color discriminationbased avoidance behavior. Society for Neuroscience Third Annual Meeting (San Diego, California), p. 130.

HYDÉN, H. 1959. Biochemical changes in glial cells and nerve cells at varying activity. In: Biochemistry of the Central Nervous System (Fourth International Congress of Biochemistry, Vienna) p. 64. Pergamon Press, New York.

HYDÉN, H. 1967. Behavioral, neural function and RNA. Prog. Nucleic Acid Res. Mol. Biol. 6

KATZ, J. J. and W. C. HALSTEAD 1950. Protein organization and mental function. Comp. Psychol. Monog. 20:1-38.

LANDAUER, T. K. 1964. Two hypotheses concerning the biochemical basis of memory. Psychol. Rev. 71:167-179.

LASHLEY, K. S. 1950. In search of the engram. In: Physiological Mechanisms in Animal Behavior (Society of Experimental Biology Symposium No. 4). Pp. 454-482. Cambridge University Press.

LIPMANN, F. 1971. Gramicidin and tyrocidine biosynthesis: a primitive process of sequential addition of amino acids on polyenzymes. In: Chemical Evolution and the Origin of Life (Buvet, R. and C. Ponnamperuma, eds.) Pp. 381-391. North-Holland Publishing Company, Amsterdam.

MCCONNELI, J. V. 1965. A tape recorder theory of memory. Worm Runner's Digest $\underline{7}(2): 3-10$.

MALIN, D. H. and H. N. GUTTMAN 1972. Synthetic rat scotophobin induces dark avoidance in mice. Science 178:12191220 .

MEISTER, A. 1973. On the enzymology of amino acid transport. Science 180:33-39.

MOSCONA, A. A. 1963. Studies on cell aggregation: demostrations of materials with selective cell-binding activity. Proc. Nat. Acad. Sci. U.S. 49:742-747.

MÜLLER, J. 1826. Zur vergleichenden Physiologie des Gesichtsinnes des Menschen und der Thiere. Knobloch, Leipzig.

REICHELT, K. L. and E. KVAMME 1973. Histamine-dependent formation of $\mathrm{N}$-acetyl-aspartyl peptides in mouse brain. J. Neurochem. 21:849-859.

REICHLIN, S. and M. MITNICK 1973. Biosynthesis of hypothalamic hypophysiotropic factors.

In: Frontiers in Neuro- 
endocrinology (Ganong, W. F. and L. Martini, eds.) Pp. 61-88. Oxford University Press, New York.

ROBERTS, R. B. and L. B. FLEXNER 1966. A model for the development of retina-cortex connections. Amer. Scientist 54: 174-183.

ROBINSON, C. E. 1966. A chemical model of long-term memory and recall. In: Molecular Basis of Some Aspects of Mental Activity (Walaas, 0., ed.) Vol. 1, Pp. 29-35, Academic Press, New York.

ROSENBLATT, F. 1967. Recent work on theoretical models of biological memory. In: Computer and Information Sciences (Tou, J., ed.) Vol. 2, Pp. 35-56. Spartan Books, Washington, D. C.

SHASHOUA, V. E. 1968. RNA changes in goldfish brain during learning. Nature 217:238-240.

SPERRY, R. W. 1963. Chemoaffinity in the orderly growth of nerve fiber patterns and connections. Proc. Nat. Acad. Sci. U.S. 50:703-710.

SQUIRE, L. R. and S. H. BARONDES 1972. Inhibitors of cerebral protein or RNA synthesis and memory. In: Macromolecules and Behavior (Gaito, J., ed.) Pp. 61-82. Appleton-Century-Crofts, New York.

SZILARD, L. 1964. On memory and recall. Proc. Nat. Acad. Sci. U.S. 51:1092-1099.

THINES, G., G. F. DOMAGK and E. SCHONNE 1973. The effect of synthetic scotophobin on the light tolerance of teleosts (Carassius auratus and Tinea tinca). In: Memory and Transfer of Information (Zippel, H. P., ed.) Pp. 363371. Plenum Press, New York.

UNGAR, G. 1968. Molecular mechanisms in learning. Perspectives Biol. Med. 11:217-232.

UNGAR, G. 1970. Molecular mechanisms in information processing. Int. Rev. Neurobiol. 13:223-253.

UNGAR, G. 1971. Chemical transfer of acquired information. In: Methods in Pharmacology (Schwartz, A., ed.) Vol. 1, Pp. 479-513. Appleton-Century-Crofts, New York.

UNGAR, G. 1972. Molecular organization of neural information processing. In: The Structure and Function of Nervous Tissue (Bourne, G. H., ed.) Vol. 4, Pp. 215-247. Academic Press, New York.

UNGAR, G. 1973. The problem of molecular coding of neural information. A critical review. Naturwissenschaften 60: $307-312$. 
UNGAR, G. and S. R. BURZYNSKI 1973. Isolation and purification of a habituation-inducing peptide from trained rat brain. Fed. Proc. 32:367Abs.

UNGAR, G. and G. CHAPOUTHIER 1971. Mécanismes moléculaires de l'utilisation de l'information par le cerveau. L'Année Psychologique 71:153-183.

UNGAR, G. , D. M. DESIDERIO and W. PARR 1972. Isolation, identification and synthesis of a specific-behaviourinducing brain peptide. Nature 238:198-202.

UNGAR, G., L. GALVAN and G. CHAPOUTHIER 1972. Possible chemical coding of color discrimination in goldfish brain. Experientia 28:1026-1027.

UNGAR, G. , L. GALVAN and R. H. CLARK 1968. Chemical transfer of learned fear. Nature 217:1259-1261.

UNGAR, G. and C. OCEGUERA-NAVARRO 1965. Transfer of habituation by material extracted from brain. Nature 207: 301-302.

ZIPPEL, H. P., ed. 1973. Memory and Transfer of Information. Plenum Press, New York. 582 pp.

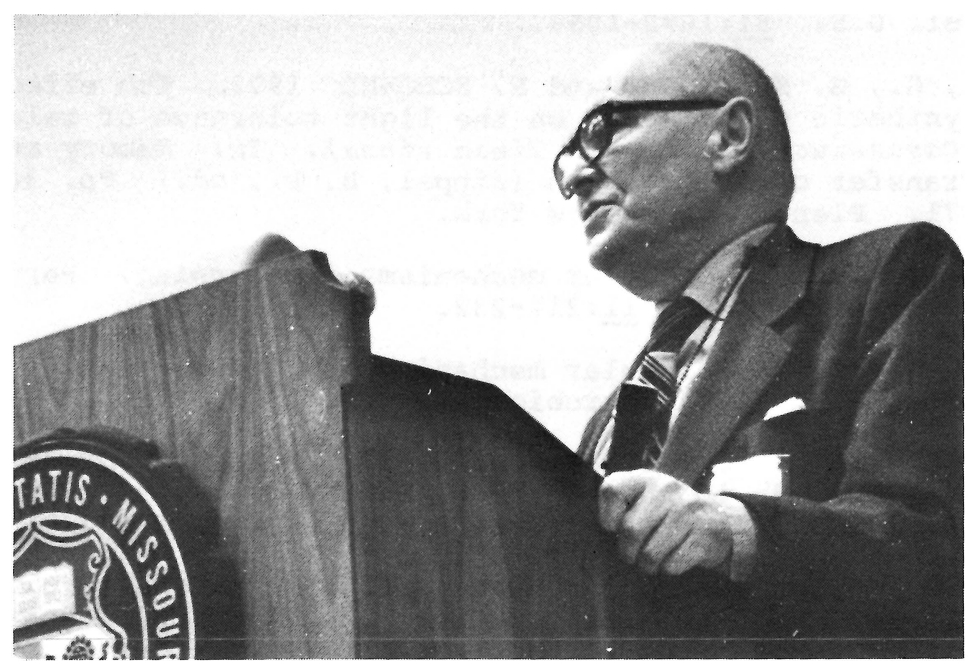

Dr. Georges Ungar lecturing 